\title{
High Angular Resolution Mid-IR Astronomy at Concordia
}

\author{
Marco Ferrari-Toniolo \\ C.N.R. - Istituto Astrofisica Spaziale e Fisica Cosmica (IASF)
}

If we concentrate our attention on the study and the evolution of star forming processes rather than on the large scale structure, from the recent use of Large Telescopes in this context there are many examples of great interest. These include the study of the early phases of aggregation of matter (stellar and planet formation), and the late phases of disaggregation during stellar evolution. In Fig 1, a spectacular example is shown of a high resolution image of an edge-on circumstellar disk of a young star (HR4796A), with respect to previous less resolved information (C.Telesco et al, Proc SPIE 4834, 101, 2002). A recent hypothesis of a Large Infrared Telescope called GTA (Grande Telescopio Antartico) has been proposed to the PNRA (Italian Plan for Antarctic Researches). This will require the following characteristics: high angular resolution in the mid-IR domain (large aperture); very high sensitivity (mainly due to the cold, dry conditions of the site); extreme simplicity in design and operational modes. The telescope will be a survey instrument and will be used almost without human intervention. The project examines the construction of a third tower at Dome $\mathrm{C}$ with the telescope configured to work in quasi-drift-scan without moving the enclosure and with a limited tracking time. In Fig 1, a sketch of the tower hosting the telescope is shown (M.F-T. et al, Proc SPIE 4836, 165, 2002). A study will be developed to determine the optimum configurations for observing in different bands, from the optical to the sub- $\mathrm{mm}$ range, with a refurbishment of the telescope and instrumentation during the summer break.

We expect there to be great gains in sensitivity from the GTA with respect to the new class of Large Telescopes operating with similar angular resolution; more detailed studies can be accomplished by the large 'temperate' Telescopes by using more sophisticated instrumentation (spectrometers, polarimeters, coronographs etc.). Even more interesting could be the use of GTA in conjunction with the LBT or other facilities for ground-based interferometry, or with respect to the ultra-high sensitivity images expected from the future IR space missionsthe GTA data will be of great help for addressing and analyzing both the space and the interferometric data. The GTA seems to be also an unavoidable step for future ELT projects.
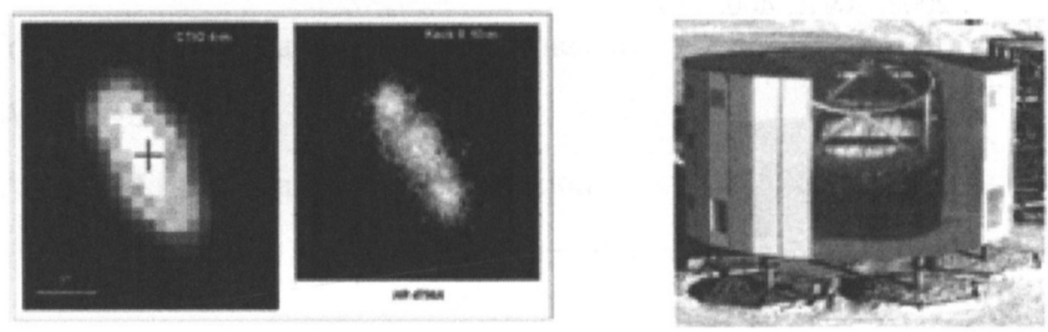